\title{
Effects of a Mixed Nutraceutical Beverage on Performance of Moderately Strenuous Aerobic Exercise Lasting Under an Hour
}

\author{
Robert A. DiSilvestro, ${ }^{1, *}$, Elizabeth Joseph ${ }^{1}$, Stacey Hart ${ }^{2}$ and Carmen B. Swain ${ }^{2}$ \\ ${ }^{1}$ Human Nutrition and ${ }^{2}$ Health and Exercise Science, The Ohio State University, 345 Campbell Hall, 1787 Neil Ave., \\ Columbus, OH 43210-1295
}

\begin{abstract}
Intake of sugar containing drinks can aid performance in long aerobic exercise sessions. On the other hand, the effects on shorter sessions are less well characterized. The present study examined whether an effect could be seen in a shorter session when a nutraceutical beverage of carbohydrates (glucose, isomaltose, waxy maize) were combined with a small amount of protein plus nutritional antioxidants ( $\beta$-carotene, vitamin $\mathrm{C}$ and vitamin $\mathrm{E})$. Recreationally trained, young adults performed two aerobic exercise testings, 4 weeks apart ( 3 mile run time + distance in 25 minutes of stationary biking + a 90 second step test). At the second testing, subjects drank $6.5 \mathrm{oz}$ of either placebo $(\mathrm{N}=20)$ or the nutraceutical beverage $(\mathrm{N}=20)$ before and after the run, and midway through the cycling. Neither beverage affected run time but the nutraceutical beverage increased biking distance and step number $(p<0.001$ for bike distance, $p<0.01$ for steps, paired $\mathrm{t}$-test). The changes for each of the latter two assessments differed significantly between beverages $(\mathrm{p}<0.01$, unpaired $\mathrm{t}$-test). In conclusion, acute use of a nutritional drink improved performance in middle + later stages of aerobic exercise lasting under 1 hour. Future work can establish an optimal ingredient mix.
\end{abstract}

Keywords: Nutraceutical, aerobic exercise, running, step test, stationary biking.

\section{INTRODUCTION}

Sugar containing beverages can improve physical functioning in moderate exercise lasting at least $2 \mathrm{~h}$, as well as for strenuous exercise lasting beyond an hour [rev in 1]. Such exercise sessions are not necessarily typical for most people who train for recreation/fitness. For example, the American College of Sports Medicine recommends $30 \mathrm{~min}$ sessions of moderately strenuous aerobic exercise [2]. Similarly, research studies often define "recreationally trained" aerobic exercisers as doing aerobic exercise $\geq 3 \mathrm{~h} / \mathrm{wk}$ [ie 3, 4]. If the subjects trained 4 to 6 times per wk, then $3 \mathrm{~h} / \mathrm{wk}$ translates to 30-45 min per session. Therefore, it seems safe to say that many people regularly do moderately intense aerobic exercise for under $1 \mathrm{~h}$. Since sugar based sports drinks have not been targeted to this type of exercise, it would be useful to know if performance of such type of exercise can be improved by a beverage with sugar + other nutritional factors.

The present study looked for improved performance using a commercial beverage containing a combination of nutraceutical ingredients. The exercise testing consisted of a sustained exercise familiar to the particular subjects (a 3 mile run), followed by a sustained exercise unfamiliar to the particular subjects ( $25 \mathrm{~min}$ of stationary bicycling), followed by a short fatiguing exercise ( $90 \mathrm{sec}$ step test). The familiar exercise was included because most people who train for general fitness have some repetition in their workouts.

*Address correspondence to this author at the Human Nutrition, The Ohio State University, 345 Campbell Hall, 1787 Neil Ave., Columbus, OH 43210-1295; Tel: 614-292-6848; Fax: 614-292-8880;

E-mails: disilvestro.1@osu.edu, RDiSilvestro@ehe.osu.edu
The unfamiliar exercise was done after a subject was somewhat fatigued. This was included because most exercisers occasionally push themselves beyond what they are used to doing. The step test was included to test acute recovery and muscle capacity reserve at the end of a work out.

\section{MATERIALS AND METHODS}

The research protocol was approved by the Ohio State University Human Subjects Biomedical Institutional Review Board. All participants signed informed consent forms. Subjects were males and females. Inclusion criteria were: age 18-30, recreationally-aerobically trained (aerobic exercise training at least 120-180 min per week for at least $6 \mathrm{mo}$ ), and having the aerobic training including runs of 3 miles or more twice per week. Exclusion criteria were: being a competitive athlete, injuries that restricted the ability to do aerobic exercise, acute major health problems or chronic conditions that affect energy metabolism or oxidant stress (ie diabetes and cancer), and cigarette smoking. All of these inclusion/ exclusion criteria were evaluated based on answers to questions on an eligibility questionnaire. Subject recruitment was done using an advertisement that specifically mentioned most inclusion/exclusion criteria. Thus, most people who filled out an eligibility questionnaire did qualify.

Subjects underwent an exercise performance test without any intervention, and then repeated the test 4 weeks later with one of two randomly assigned, acute interventions, Placebo (Crystal Light ${ }^{\circledR}$ ) or the nutraceutical beverage (Endurance Fuel ${ }^{\mathrm{TM}}$ from Twinlab ${ }^{\circledR}$, contents in Table 1). The subjects picked out a paper with a number on it that was randomly assigned by computer to one of the intervention products. The subjects did not know which product they received. 
Table 1. Composition of the Nutraceutical Beverage. (Endurance $\mathrm{Fuel}^{\mathrm{TM}}$ from Twinlab $\left.{ }^{\circledR}\right)$

\begin{tabular}{|l|l|}
\hline Beta Alanine & $8.0 \%$ \\
\hline Maltodextrin & $16.4 \%$ \\
\hline Iso-maltulose & $16.4 \%$ \\
\hline Waxy Maize & $16.4 \%$ \\
\hline Glucose & $16.0 \%$ \\
\hline Calcium Caseinate $90 \%$ & $9.4 \%$ \\
\hline Whey Protein Isolate 90\% & $9.4 \%$ \\
\hline Natural flavor & $2.6 \%$ \\
\hline Citric acid & $2.3 \%$ \\
\hline Sodium chloride & $0.8 \%$ \\
\hline Vitamin blend, sweeteners & $2.3 \% *$ \\
\hline $\begin{array}{l}\text { Values are in } \% \text { by weight of dry powder. The exercise sessions used } \\
\text { 30g (see Methods text for details) } \\
\text { *Vitamins provided per exercise session: } 2500 \text { IU vitamin A as } \\
\text { beta-carotene, 60 mg vitamin C as ascorbic acid, 30 IU vitamin E as } \\
\text { d-alpha tocopherol acetate. }\end{array}$
\end{tabular}

The exercise testing consisted of the following:

- Time for a 3 mile run (a familiar activity for this study's subjects)

- Distance covered in 25 min of stationary cycling (an unfamiliar activity for this study's subjects); this was done after post-run walking for $1 / 8$ of a mile in about $1 \mathrm{~min} 30$ sec; the cycling test was carried out with the machine set in the manual mode using a resistance level determined on a day prior to the testing; the resistance level was set where a subject could do $5 \mathrm{~min}$ at $80 \mathrm{rpm}$ with a moderate rating of perceived exertion. If during the test, the subject felt the resistance setting was too hard or too easy, it was changed once. During the second testing, the times at each resistance were repeated.

- A 90 sec step test done about 1.5 min after the cycling (a test of acute recovery); subjects walked during the 1.5 min recovery; step test results were total steps completed with alternating lead legs; step test was completed on a permanent seating area near the running track (step height of $41 \mathrm{~cm}$ ).

For the first exercise testing, subjects were provided water just before the run, just after the post-run walk, and midway through the cycling. For the second intervention, subjects were provided about $6.5 \mathrm{oz}$ of the intervention beverage or placebo at the same times.

For statistical analysis, significance was set at $\mathrm{p}<0.05$. For each measure, for each of the two treatment groups (placebo and nutraceutical beverage), session 1 values were compared to session 2 values by paired Student's t-test. This determined if each of the treatments had any significant effects. For further statistical analysis, the results for each measure were expressed as the change in values (session 2 value - session 1 value). The changes in the nutraceutical beverage group were compared to the changes in placebo by unpaired Student's t-test.

\section{RESULTS}

Certain subject characteristics are noted in Table 2. Male and female data were pooled since no differences in trends were noted. The 3 mile run time was not significantly affected by either placebo or the nutraceutical beverage (Table 3). The nutraceutical beverage, but not the placebo, increased the biking distance per $25 \mathrm{~min}$ to a statistically significant degree (Fig. 1).

Table 2. Selected Subject Characteristics

\begin{tabular}{|l|l|l|}
\hline Variable & Placebo & Nutraceutical Beverage \\
\hline \hline $\mathrm{N}$ & 22 & 22 \\
\hline Age (mean $\pm \mathrm{SD})$ & $21 \pm 3$ & $21 \pm 3$ \\
\hline 3 mile run time (mean $\pm \mathrm{SD})$ & $24.3 \pm 1.0$ & $23.4 \pm 0.5$ \\
\hline
\end{tabular}

No significant difference for age or initial run time ( $\mathrm{p}>0.05$, unpaired $\mathrm{t}$-test).

Table 3. Times for 3 Mile Runs

\begin{tabular}{|l|l|l|}
\hline Variable & Placebo & Nutraceutical Beverage \\
\hline \hline Trial 1 & $24.3 \pm 1.0$ & $23.4 \pm 0.5$ \\
\hline Trial 2 & $23.8 \pm 0.9$ & $23.1 \pm 0.5$ \\
\hline
\end{tabular}

Run times are means of minutes + SEM

$p>0.05$ for each treatment by paired t-test.

\section{Bike Distance}

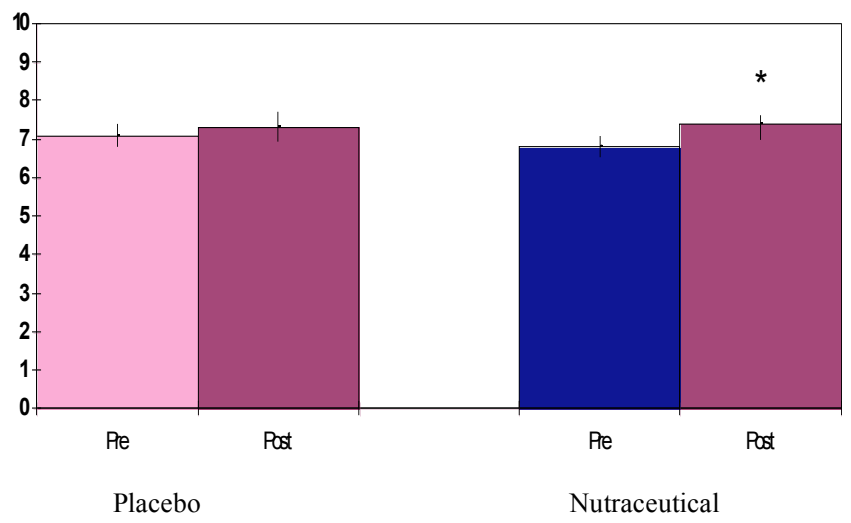

Fig. (1). Bike distances in mile means \pm SEM *p $<0.001$, paired t-test.

Similarly, the nutraceutical beverage, but not the placebo, increased the number of steps per $90 \mathrm{sec}$ to a statistically significant degree (Fig. 2). If the biking distance and step results were expressed as the differences between pre- and post-intervention, the changes in the biking and step tests for the nutraceutical beverage was significantly different from placebo (Fig. 3).

Power calculations were done to test the hypothesis that for each exercise evaluation, placebo and the nutraceutical beverage do not show differences for the change in values from the first to second trial. Using the data from Fig. (3), a power value of about $100 \%$ was obtained for a two tailed test, $\mathrm{p}<0.01,22$ subjects. For the run time, the data used for Table $\mathbf{2}$ was recalculated to derive a change and variance 
Step Test

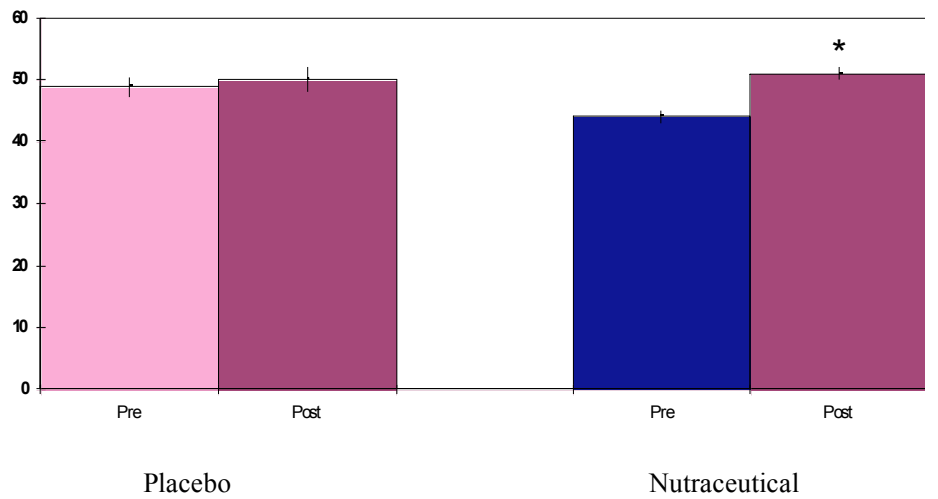

Fig. (2). Step tests in mean steps $/ 90 \sec \pm \operatorname{SEM} * \mathrm{p}<0.01$, paired t-test.

Changes in Biking Distance \& Step Test

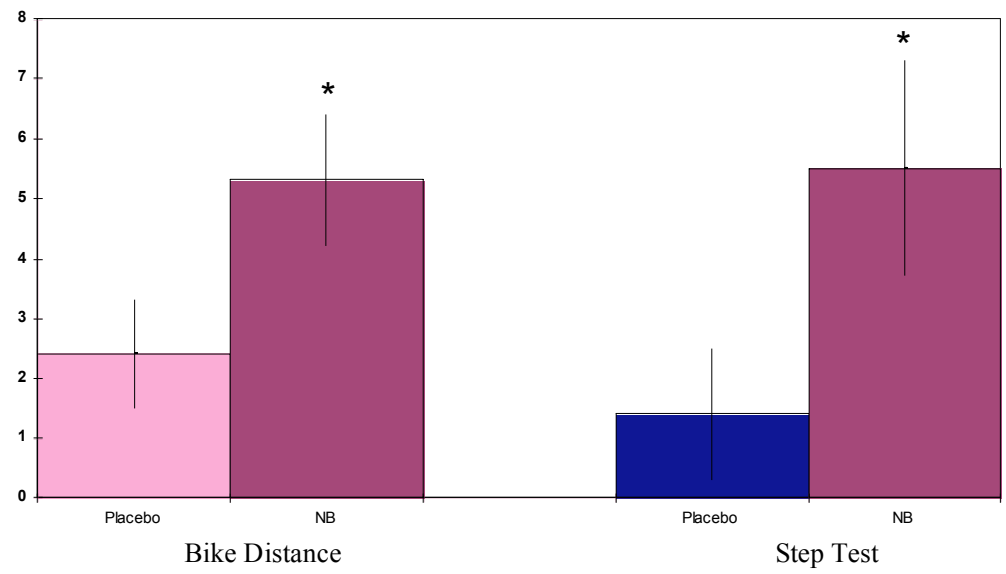

Fig. (3). Change in biking distance $(1 / 10$ miles \pm SEM) \& step test ( steps \pm SEM); NB $=$ Nutraceutical beverage *p $<0.01$, unpaired t-test.

for trial 1 versus trial 2. For the nutraceutical beverage, the change was altered to give a minimal desired change of 15 seconds more than the placebo. For this change, a power value of about $84 \%$ was obtained for a two tailed test, $\mathrm{p}<0.05,22$ subjects.

\section{DISCUSSION}

Previous work has shown that sugar-containing beverages can improve physical functioning in moderate exercise lasting at least $2 \mathrm{~h}$, as well as for strenuous exercise lasting beyond an hour [rev in 1]. An assumption has arisen that acute nutritional beverage intake will not affect aerobic exercise lasting under $1 \mathrm{~h}$. However, this assumption may be based more on a lack of testing than on negative results. The present study demonstrated that for moderately intense sessions lasting under $1 \mathrm{~h}$, intake of a nutraceutical beverage can improve performance. The particular session examined here brought the subjects to three fatigue points. The results obtained for this exercise session may or may not apply to other types of aerobic exercise lasting about the same time.

The nutraceutical beverage did not affect the 3 mile run phase of the exercise testing. This lack of effect may have derived more from the stage of the exercise testing rather than a lack of effect on running per se. Although the running required exertion, it was a medium length, aerobic activity familiar to the exercisers. Also, it was initiated while the exercisers were fresh. Thus, in this test population, for the run, the nutraceutical beverage did not affect anti-fatigue mechanisms beyond what was already accomplished by previous exercise training and diet. In contrast, after the subjects came to one fatigue point (post-run), briefly recovered, then resumed with a different exercise, stationary biking, the nutraceutical beverage aided performance. The same was true for the step test, which was a measure of acute recovery after sustained exertion. Thus, depletion of some body chemicals may need to occur before the beverage could exert an effect, though no measures of any specific depletions were made in this initial study.

The $90 \mathrm{sec}$ step test done here represented the end of an aerobic exercise session with a fairly high degree of anaerobic exertion. This may have been the most likely part of the session for some of the nutraceutical ingredients to give an effect. For example, the anaerobic respiration occurring during the step test could make the ingested carbohydrates useful for quick glucose supply to the muscles. 
Performance of the biking phase of the exercise test was also affected by the nutraceutical beverage. At the biking stage, the subjects had already reached one fatigue point, and then had to sustain an unfamiliar exertion for $25 \mathrm{~min}$. Thus, they tended to do the biking phase at a steady, but moderate pace. Therefore, anaerobic respiration would not peak as high as for the step test. So, the anti-fatigue mechanisms for the beverage in the biking phase may have been partly different from what occurred at the step test.

It is not certain which components of the nutraceutical beverage had the most effect, or whether all components were necessary to see an effect. The rationale for each ingredient is as follows:

\section{Isomaltose and Waxy Maize}

This disaccharide and starch digest slowly with a smaller insulin response than caused by sucrose $[5,6]$; these actions may maintain steady glucose availability for muscle. The waxy maize also has a low osmality, which can enhance gastric emptying [7], which may allow this starch to stay in the intestine, supply a steady stream of glucose for the blood, but not divert a lot of blood flow away from the muscles to the stomach. One study [8] has shown that consuming waxy maize before exercise can improve performance in a very long cycling bout, but this carbohydrate had not been tested in the type of exercise used in the present study.

\section{Casein and Whey Proteins}

Intake of protein along with carbohydrate has been proposed to slow glycogen depletion; this has been reported to reduce fatigue in one protocol for very long intense cycling exercise [9], though it did not work well in another protocol for a long cycling exercise [10]. One difference between the two studies is that the first [9] used varying intensities while in the second [10], a long constant effort preceded a testing to fatigue. The current study exercise protocol, though much shorter than for either of these cycling studies, resembles the first protocol in that subjects varied exercise pace at different stages, reached fatigue more than once, then partially recovered. This study uses a mixture of two complete, but different proteins since it is not known if any one type of protein would work best.

\section{Glucose}

This sugar, or sucrose or maltodextrin, which contains glucose, is the starting point for carbohydrate containing drinks that are to be taken during exercise. Glucose and maltodextrin may compliment the other carbohydrates of this study by providing a fast absorbed sugar to mix with the slow absorbed sugars. Although the absorption of glucose may be slowed by the other carbohydrates and the protein in the study beverage, glucose could still provide more blood sugar early in the exercise than the other carbohydrates.

\section{Beta Carotene, Vitamin $\mathrm{C}$ and Vitamin $\mathrm{E}$}

These agents can exert complementary antioxidant actions [11]. Reactive oxidant molecules, which either are free radicals or produce free radicals, are generated during exercise and can accelerate fatigue [12, 13]. Numerous studies have shown than acute exercise produces oxygen and nitro- gen based reactive molecules as evidenced by accumulation of tissue products of oxidant damage [rev in 13]. A review article by Jackson [14] proposes that the reactive species are produced by exercise via multiple processes such as mitochondrial respiration, prostanoid metabolism, autooxidation of catecholamines, and the activities of certain enzymes such as NADPH oxidase and xanthine oxidase. Also, serum myeloperoxidase activity, which is both a pro-oxidant in itself and marks activation of pro-oxidant neutrophils, increases during aerobic exercise [ie 15].

\section{$\beta$-alanine}

The commercial product contained this compound, though it is not certain that it would contribute to any effects. Intake of $\beta$-alanine for at least 4 weeks can increase muscle carnosine, which can affect muscle performance [16], but no evidence exists that a single intake of $\beta$-alanine has such effects.

As stated above, it is not certain the degree to which each of the ingredients contributed to the effects seen. However, identification of the exact contribution of each ingredient was not the main point of this study. Instead, this study established that for moderately strenuous aerobic exercise lasting under $1 \mathrm{~h}$, with 3 different fatigue points, performance can be enhanced by acute intake of a nutritional beverage.

\section{CONCLUSION}

It was shown that acute intake of a nutritional beverage during exercise can affect performance in moderately strenuous aerobic exercise lasting under $1 \mathrm{~h}$. This type of exercise is often done by people who train to a moderately high fitness level, but who are not competitive athletes. Future work can look at how different variations of nutritional ingredients affect performance of this type of exercise.

\section{ACKNOWLEDGEMENTS}

This study was supported by a grant to Robert DiSilvestro by Ideasphere Inc. The authors gratefully acknowledge fine assistance with the exercise testing from Trisha Marshall, David DiSilvestro, Amanda O'Dell, Wei Feng, and Lisa DiSilvestro.

The author(s) declare that they have no competing interests.

\section{REFERENCES}

[1] Jeukendrup AE. Carbohydrate intake during exercise and performance. Nutrition 2004; 20: 669-77.

[2] Available in: http://www.acsm.org/AM/Template.cfm? Section= Home Page\&TEMPLATE $=$

CM/HTMLDisplay.cfm\&CONTENTID $=7764$.

[3] Sinclair LM, Hinton PS. Prevalence of iron deficiency with and without anemia in recreationally active men and women. J Am Diet Assoc 2005; 105: 975-8.

[4] Popovic M, Puchner S, Endler G, Foraschik C, Minar E, Bucek RA. The effects of endurance and recreational exercise on subclinical evidence of atherosclerosis in young adults. Am J Med Sci 2010; 339: 332-6.

[5] Achten J, Jentjens RL, Brouns F, Jeukendrup AE. Exogenous oxidation of isomaltulose is lower than that of sucrose during exercise in men. J Nutr 2007; 137: 1143-8.

[6] Sands AL, Leidy HJ, Hamaker BR, Maguire P, Campbell WW. Consumption of the slow-digesting waxy maize starch leads to blunted plasma glucose and insulin response but does not influence 
energy expenditure or appetite in humans. Nutr Res 2009; 29: 383-90.

[7] Vist GE, Maughan RJ. Gastric emptying of ingested solutions in man: effect of beverage glucose concentration. Med Sci Sports Exerc 1994; 26: 1269-73.

[8] Goodpaster BH, Costill DL, Fink WJ, et al. The effects of preexercise starch ingestion on endurance performance. Int J Sports Med 1996; 17: 366-72.

[9] Ivy JL, Res PT, Sprague RC, Widzer MO. Effect of a carbohydrateprotein supplement on endurance performance during exercise of varying intensity. Int J Sport Nutr Exerc Metab 2003; 13: 382-95.

[10] Osterberg KL, Zachwieja JJ, Smith JW. Carbohydrate and carbohydrate + protein for cycling time-trial performance. J Sports Sci 2008; 26: 227-33.

[11] Wardlaw GM, Hampl JS, DiSilvestro RA. Perspectives in Nutrition. 6th ed. New York USA: McGraw Hill 2004.
[12] Reid MB. Free radicals and muscle fatigue: Of ROS, canaries, and the IOC. Free Radic Biol Med 2008; 44: 169-79.

[13] Fisher-Wellman K, Bloomer RJ. Acute exercise and oxidative stress: a 30 year history. Dyn Med 2009; 8: 1-26.

[14] Jackson MJ, Sen C, Packer L, Hanninen O, Eds. Exercise and oxygen radical production by muscle. In Handbook of oxidants and antioxidants in exercise. Amsterdam: Elsevier Science 2000; 57-68.

[15] Rossi AL, Blostein-Fujii A, DiSilvestro RA. Soy beverage consumption by young men: increased plasma total antioxidant status and decreased acute, exercise-induced muscle damage. J Nutraceuticals Funct Med Foods 2000; 3: 33-44.

[16] Derave W, Everaert I, Beeckman S, Baguet A. Muscle carnosine metabolism and beta-alanine supplementation in relation to exercise and training. Sports Med 2010; 40: 247-63.

(C) DiSilvestro et al.; Licensee Bentham Open.

This is an open access article licensed under the terms of the Creative Commons Attribution Non-Commercial License (http://creativecommons. org/licenses/ by-nc/3. 0/) which permits unrestricted, non-commercial use, distribution and reproduction in any medium, provided the work is properly cited. 